\title{
Novel withanolides target medullary thyroid cancer through inhibition of both RET phosphorylation and the mammalian target of rapamycin pathway
}

\author{
Abbas K. Samadi, PhDa, Joseph Bazzill, BS ${ }^{a}$, Xuan Zhang, PhD ${ }^{a}$, Rob Gallagher, PhD ${ }^{b}$, \\ Hauping Zhang, $\mathbf{P h D}^{\mathrm{b}}$, Rao Gollapudi, $\mathbf{P h D}^{\mathrm{b}}$, Kelly Kindscher, $\mathbf{P h D}^{\mathrm{b}}$, Barbara Timmermann, \\ PhD $^{\mathrm{b}}$, and Mark S. Cohen, MD, FACS ${ }^{\mathrm{c}}$ \\ aDepartment of Surgery, University of Kansas Medical Center, Kansas City \\ bDepartment of Medicinal Chemistry, The University of Kansas, Lawrence, KS \\ 'Department of Surgery, University of Michigan Hospital and Health Systems, Ann Arbor, MI
}

\begin{abstract}
Background-Despite development of current targeted therapies for medullary thyroid cancer (MTC), long-term survival remains unchanged. Recently isolated novel withanolide compounds from Solanaceae physalis are highly potent against MTCs. We hypothesize that these withanolides uniquely inhibit RET phosphorylation and the mammalian target of rapamycin (mTOR) pathway in MTC cells as a mechanism of antiproliferation and apoptosis.
\end{abstract}

Methods-MTC cells were treated with novel withanolides and MTC-targeted drugs. In vitro studies assessed cell viability and proliferation (MTS; trypan blue assays), apoptosis (flow cytometry with Annexin V/PI staining; confirmed by Western blot analysis), long-term cytotoxic effects (clonogenic assay), and suppression of key regulatory proteins such as RET, Akt, and mTOR (by Western blot analysis).

Results-The novel withanolides potently reduced MTC cell viability (half maximal inhibitory concentration $\left[\mathrm{IC}_{50}\right], 270-2,850 \mathrm{nmol} / \mathrm{L} ; 250-1,380 \mathrm{nmol} / \mathrm{L}$ for vandetanib; $360-1,640 \mathrm{nmol} / \mathrm{L}$ for cabozantinib) with induction of apoptosis at $<1,000 \mathrm{nmol} / \mathrm{L}$ of drug. Unique from other targeted therapies, withanolides suppressed RET and Akt phosphorylation and protein expression (in a concentration- and time-dependent manner) as well as mTOR activity and translational activity of $4 \mathrm{E}-\mathrm{BP} 1$ and protein synthesis mediated by $\mathrm{p} 70$ S6kinase activation at $\mathrm{IC}_{50}$ concentrations.

Conclusion-Novel withanolides from Physalis selectively and potently inhibit MTC cells in vitro. Unlike other MTC-targeted therapies, these compounds uniquely inhibit both RET kinase activity and the Akt/mTOR prosurvival pathway. Further translational studies are warranted to evaluate their clinical potential.

(C) 2012 Mosby, Inc. All rights reserved.

Reprint requests: Mark S. Cohen, MD, FACS, Associate Professor, Director of Endocrine Surgery Research, Division of Endocrine Surgery, Department of Surgery, 2920K Taubman Center SPC 5331, University of Michigan Hospital and Health Systems, 1500 E. Medical Center Dr., Ann Arbor, MI 48109-5331. cohenmar@med.umich.edu. 
Although the incidence of medullary thyroid cancer (MTC) has grown each year, recently $>2,200$ new cases reported in the United States in $2010,{ }^{1}$ survival statistics in this disease have not significantly improved over the last 3 decades, warranting novel therapies with better efficacy. For early stage disease confined to the thyroid or locoregional nodes, 5-year disease-specific survival rates have been reported at $>80 \%$; however, once tumors metastasize systemically, survival drops significantly to $\langle 55 \%$. 2,3 Operative resection is the standard of care for local disease and recurrence; however, $>50 \%$ of patients recur or progress despite optimal operative resection. ${ }^{2}$

Standard chemotherapy regimens have limited efficacy in poorly differentiated thyroid cancers such as MTC. Response rates are often temporary and occur in $<10-20 \%$ of patients and are without long-term benefit. Additionally, these drugs carry systemic toxicities that can be dose limiting. ${ }^{4}$ Recently, targeted therapies developed on the genetic and molecular basis of MTCs have led to several Phase I, II, and III human trials worldwide. Because a majority of MTCs are owing to mutations of the RET protooncogene (which encodes a transmembrane tyrosine kinase receptor), ${ }^{3,5,6}$ a number of tyrosine kinase inhibitors (TKI) and multikinase inhibitors have been developed in the last decade, including RET-kinasespecific inhibitors that share the property of binding to the RET/ATP-binding pocket. These TKIs that target RET include drugs such as vandetanib, sorafenib, sunitinib, imatinib, axitinib, motesanib, gefitinib, and cabozantinib (XL184). ${ }^{7-9}$ Although these drugs may stabilize disease progression in many patients, they lack durable, long-term responses and carry moderate systemic toxicity for many patients. Opportunities, therefore, remain to identify novel durable therapies for advanced MTC.

Natural products continue to play a highly significant role in the drug discovery and development process, particularly in the area of cancer therapeutics, where a majority of the most widely used cytotoxic agents are of natural origin. Several genera in the large plant family Solanaceae produce compounds called withanolides, of which the most important and well-described is the steroidal lactone, withaferin A (WA). ${ }^{10,11}$ The withanolides exert a number of different effects including anti-stress, anti-inflammatory, and cytotoxic activities; however, their role as anticancer agents in MTC is currently being investigated. ${ }^{12,13}$

WA isolated from the Withania somnifera plant has been shown to significantly inhibit MTC tumor mass in vivo in a metastatic mouse model. ${ }^{14,15}$ In addition to induction of apoptosis and inhibition of the PI3kinase/Akt pathway by WA in thyroid cancers, WA has also been reported to inhibit Notch signaling and the mammalian target of rapamycin (mTOR) pathway in colon cancer cells. ${ }^{16}$ In the present study, the anti-cancer activity of several novel withanolides will be examined in MTC cells and compared with WA and TKIs used in MTC clinical trials such as 17-AAG, axitinib, vandetanib, and cabozantinib. We hypothesize that novel withanolide derivatives, unlike other targeted kinase inhibitors, uniquely inhibit MTC cell proliferation and induce apoptosis through a combination of RET kinase inhibition as well as inhibition of the Akt/mTOR signaling pathway. 


\section{MATERIALS AND METHODS}

\section{Reagents and cell culture}

All chemical reagents were obtained from Sigma Chemical Co (St. Louis, MO) unless specified otherwise. Antibodies to phospho-Thr-389 S6K, phospho-Thr-36/47 4E-BP1, phospho-Ser-65 4E-BP1, 4E-BP1, phospho-mTOR, mTOR, phospho-Akt, Akt, phosphoERK, ERK, phospho-p70S6K, p70S6K, caspase 3, and PARP from Cell Signaling Technology (Beverly, MA); antibodies to actin from Millipore (Temecula, CA), and horseradish peroxidase-labeled anti-mouse, anti-goat, and anti-rabbit secondary antibodies from Santa Cruz Biotechnology (Santa Cruz, CA). The human MTC cell lines included MTC-TT cells (derived from MEN2A patient with codon 634 mutation) obtained from ATCC (Manassas, VA) and DRO 81-1 cells (derived from a patient with sporadic MTC) were provided by Dr Guy Juillard (University of California, Los Angeles, CA). The papillary thyroid cancer cell-line, TPC 1 (with a RET/PTC mutation), was provided by Dr Sissy M. Jhiang (Ohio State University, Columbus, OH). DRO81-1 and TPC 1 cancer cell lines were grown in RPMI 1640 medium (Sigma) supplemented with $2 \mathrm{~g} / \mathrm{L}$ sodium bicarbonate, $0.14 \mathrm{mmol} / \mathrm{L}$ nonessential amino acids, $1.4 \mathrm{mmol} / \mathrm{L}$ sodium pyruvate, and $10 \%$ fetal bovine serum at a constant temperature of $37^{\circ} \mathrm{C}$ with a humidified atmosphere of $5 \%$ carbon dioxide $\left(\mathrm{CO}_{2}\right)$. TT cells were cultured in RPMI 1640 medium supplemented with 2 $\mathrm{mmol} / \mathrm{L} \mathrm{L}$-glutamine, $1.5 \mathrm{~g} / \mathrm{L}$ sodium bicarbonate, and $15 \%$ fetal bovine serum. The cells were passaged twice a week. For growth experiments, cells were trypsinized and cells were cultured overnight before treatment. Control cultures were incubated in medium containing vehicle alone. Drug compounds utilized for these experiments included WA, the novel withanolides withalongolide A [19-hydroxy withaferin A (WGA; also referenced as compound X001), withalongolide A 4,19,27-triacetate (WGA-TA; also referenced as compound X003), withalongolide D (WGD; also referenced as compound X005), withalongolide B 4-acetate (WGB-MA; also referenced as compound X032), withalongolide B 4,19-diacetate (WGB-DA; also referenced as compound X033), all obtained from Dr Barbara Timmermann's laboratory (Lawrence, KS). Additional drugs included several TKIs used in MTC trials. Axitinib (Cat \# A-1107), vandetanib (Cat \# V-9402), and 17-AAG (Cat \# A-6880) were obtained from LC Laboratories (Woburn, MA); cabozantinib (XL184) (Cat \# S1119) was obtained from SelleckChem (Houston, TX).

\section{Cell growth assay}

Cell viability was determined by 3-(4,5-dimethylthiazol-2-yl)-5-(3carboxymethoxyphenyl)-2-(4-sulphophenyl)-2H-tetrazolium (MTS) dye-reduction assay (Promega, Madison, WI) measuring mitochondrial respiratory function according to the manufacturer's instruction. MTC cells $\left(2-5 \times 10^{3} /\right.$ well) were plated in 96-well microtitre plates and treated with drugs at various concentrations $(0.03-20 \mathrm{mmol} / \mathrm{L})$ for 72 hours. Cells were then incubated with MTS dye $(2 \mathrm{mg} / \mathrm{mL}$ per $20 \mu \mathrm{L} /$ well) for 2 hours. Absorbance was determined in a Biotek plate reader at $490 \mathrm{~nm}$. The absorbance is directly related to viable cell number. 


\section{Flow cytometry analysis}

For cell cycle studies, DRO81- 1 cells ( $10^{5}$ cells) in 60-mm plates were exposed to withanolides for 24 and 48 hours. After cells were washed, DNA was stained with $50 \mu \mathrm{g} / \mathrm{mL}$ propidium iodide (PI) solution $(0.02 \mu \mathrm{g} / \mu \mathrm{L}$ RNase A, $1.0 \mathrm{mg} / \mathrm{mL}$ sodium citrate-dihydrate, $0.1 \mu \mathrm{g} / \mu \mathrm{L}$ PI, $0.3 \%$ Triton X-100) at $25^{\circ} \mathrm{C}$ for 30 minutes. For fluorescence-activated cell sorting analysis, the FACS-can (Becton Dickinson, Franklin Lakes, NJ) was used. Cell-cycle analysis was performed with the use of ModFit LT software (Verity Software House, Inc, Topsham, ME). For Annexin V/PI studies, cells were washed after treatment and were collected by trypsinization. Cells were washed twice with $1 \times$ phosphate-buffered saline (PBS) and then stained with Annexin V-FITC and PI according to the manufacturer's instruction (BD Bioscience, San Jose, CA).

\section{Western blot analysis}

After treatment, DRO81-1 cells were lysed with ice-cold lysis buffer [ $150 \mathrm{mmol} / \mathrm{L} \mathrm{NaCl}, 40$ mmol/L HEPES (pH 7.4), 2 mmol/L EGTA, 2.5 mmol/L MgCl2, $1 \%$ Triton X-100, and $1 \mathrm{X}$ EDTA-free protease inhibitors (Sigma)]. The soluble fractions from cell lysates were isolated by centrifugation at $14,000 \mathrm{rpm}$ for 20 minutes in a microfuge. Protein concentration was determined by bicinchoninic acid protein assay kit according to the manufacturer's instructions (Pierce Biotechnology, Rockford, IL). Equivalent protein extracts $(15-50 \mathrm{mg})$ from each sample were electrophoresed on $8-15 \%$ sodium dodecyl sulfate polyacrylamide gel electrophoresis mini gels. Proteins were transferred onto Hybond nitrocellulose (Amersham, Piscataway, NJ) in a Bio-Rad Trans blot apparatus.

Nitrocellulose matrices were preblocked with 3\% nonfat milk powder in PBS and $0.05 \%$ Tween-20 for 1 hour at room temperature. After PBS-Tween washes, preblocked matrices were incubated with appropriate dilution of primary antibody. Reactive bands were visualized specific bands by enhanced chemiluminescence ECL (Pierce Biotechnology).

\section{Clonogenic assay}

DRO81-1 cells were cultured into 60-mm plates (300 cells/plate), then left in the incubator overnight to attach. Cells were treated with withanolides for 72 hours at $37^{\circ} \mathrm{C}$ in a humidified atmosphere of $95 \%$ air and $5 \% \mathrm{CO}_{2}$. After treatment media was removed and fresh media without drug was added. Cells incubated for 2 weeks to allow the formation of macroscopic colonies. Media was changed 3 times a week. The cells were then stained with Coomassie blue. At least 2 independent experiments, each using triplicate plates, were performed for each cell line. Photographic documentation was recorded.

\section{Statistical analysis}

All in vitro data points were run in triplicate and expressed as a mean values \pm standard error of the mean. Raw data were analyzed by a Student's unpaired $t$-test and Fisher exact tests using a standard statistical analysis software package (SPSS version 17.0; SPSS Inc, Chicago, IL); $P<.05$ was defined as significant. 


\section{RESULTS}

\section{Withanolides impair MTC cell growth in part through induction of apoptosis}

DRO81-1, TT-cells, and TPC1 cells were incubated with each of the drugs structurally listed in Figure 1 for 72 hours $(0.03-20 \mathrm{mmol} / \mathrm{L})$ and cell viability was determined using MTS dye reduction assay. Half maximal inhibitory concentration $\left(\mathrm{IC}_{50}\right)$ values were determined for each compound in all 3 cell-lines tested and are tabulated in the Table. Of all the compounds tested, 17-AAG was the most potent in all cell-lines with an average $\mathrm{IC}_{50}$ in MTC $18 \pm 4$ $\mathrm{nmol} / \mathrm{L}$. All of the withanolides tested demonstrated significant potency against MTC cells except for WGD, which structurally lacks the double bond in the A-ring of the molecule and results in a loss of anticancer activity (structure-activity-relationship; data not shown). The remaining withanolides demonstrated excellent potency in DRO81-1 MTC cells compared with the targeted TKIs, but were less potent than vandetanib and cabozantinib in TT cells. Addition of acetyl groups to the withalongolide A (WGA) molecule as noted with withalongolide B 4-acetate (WGB-MA; a mono-acetyl analog), withalongolide B 4,19diacetate (WGB-DA, a di-acetyl analog) and withalongolide A 4,19,27-triacetate (WGATA, a tri-acetyl analog), resulted in increased potency compared with the parent molecule WGA. This was observed in all 3 cell-lines. Comparing the tri-acetyl and mono-acetyl analogs, WGA-TA was significantly more potent than WGB-MA in each cell line tested ( $P$ $<.01)$. From this initial cell viability analysis, a ranking of potency for the withanolides from least potent to most potent would include WGD, WGA, WA, WGB-MA, WGB-DA, and WGA-TA. Owing to its stability and consistency of growth in vitro compared with TT cells, further mechanistic studies were carried out using DRO81-1 cells.

\section{Withanolides promote cell cycle arrest in MTC cells}

We next examined that the effect of novel withanolides on modulation of cell cycle progression in MTC cells. WGA induced a shift in cell cycle arrest from G0/G1 arrest to G2/M arrest in DRO81-1 cells at $6.0 \mu \mathrm{mol} / \mathrm{L}$ for 24 hours and both at 3.0 and $6.0 \mu \mathrm{mol} / \mathrm{L}$ for 48 hours treatment as noted by PI staining peaks on flow cytometry (Fig 2, A). This shift with treatment to G2/M arrest was also observed with WGB-MA and graphed quantitatively in Figure 2, $B$. After 48 hours WGA treatment at $3 \mu \mathrm{mol} / \mathrm{L}$ there was a shift in $\mathrm{G} 2 / \mathrm{M}$ arrest from $20 \%$ to $35 \%$ of cells arrested at this checkpoint, whereas at $6 \mu \mathrm{mol} / \mathrm{L}$ this shift increased to $40 \%$ of cells in $\mathrm{G} 2 / \mathrm{M}$ ( $P<.01$ compared with controls). Similarly, at 48 hours both 1 and $3 \mu \mathrm{mol} / \mathrm{L}$ WGB-MA resulted in an increase in G2/M arrest from $25 \%$ to $42 \%$ of cells arrested at this checkpoint $(P<.01)$. WGB-DA also induced G2/M cell-cycle arrest in MTC cells. After 24 hours of treatment, WGB-DA treatment induced G2/M shift from 23\% to $50 \%$ and $40 \%$ at 1 and $3 \mu \mathrm{mol} / \mathrm{L}$. Similar results were obtained with MTC cells were treated with WGB-DA for 48 hours (Fig 2, B).

\section{Withanolides induced apoptosis in MTC cells}

To explore the mechanism of withanolide-induced growth suppression, we examined the effect of these novel compounds on the induction of apoptosis in MTC cells. Initial evaluation was performed with annexin V/PI staining on flow cytometry and confirmed with caspase 3 activation and poly-ADP ribose polymerase (PARP) cleavage on Western blot analysis. WGA induced apoptosis in MTC cells at 3 and $6 \mu \mathrm{mol} / \mathrm{L}$ for 24 hours $(5.1 \%$ and 
$12.0 \%$, respectively) and 48 hours (12.2\% and 49\%, respectively; $P<.001$; Fig $3, A$ and $D$ ). Similarly WGB-MA induced apoptosis at 1 and $3 \mu \mathrm{mol} / \mathrm{L}$ in MTC cells at 24 hours (3\% and $22 \%$, respectively) and at 48 hours (28\% and 82\%, respectively; $P<.001$; Fig 3, $B$ and $D$ ). WGB-DA was the most potent withanolide for inducing apoptosis in MTC cells. At 1 and 3 $\mu \mathrm{mol} / \mathrm{L}$ WGB-DA in MTC cells at 24 hours gated $11 \%$ and $46 \%$ of cells toward apoptotic cell death, respectively, and at 48 hours this increased to $68 \%$ of cells undergoing apoptosis at $1 \mu \mathrm{mol} / \mathrm{L}$ and $83 \%$ of cells undergoing apoptosis at $3 \mu \mathrm{mol} / \mathrm{L}(P<.001 ;$ Fig $3, C$ and $D)$. To confirm these results the levels of cleaved caspase 3 and the endogenous caspase 3 substrate, PARP, were determined by Western blot analysis of cells treated for 24 hours. Fig 3, $E$ shows that WGB induced cleavage of caspase at $6 \mu \mathrm{mol} / \mathrm{L}$ concentration after 24 hours treatment, whereas the more potent withanolides WGB-MA and WGB-DA activated caspase 3 and cleaved PARP starting at $1 \mu \mathrm{mol} / \mathrm{L}$ drug levels and peaking around $3 \mu \mathrm{mol} / \mathrm{L}$ of drug. Similarly, the effect of axitinib, vandetanib- and XL184-treated DRO81-1 cells demonstrated that these TKIs slightly induced caspase activation and PARP cleavage but this occurred less robustly than observed with the novel withanolide compounds (Fig 3,E).

\section{Withanolides promote inhibition of clonogenic growth}

Clonogenic assay was performed to determine the long-term toxicity effect of withanolides on MTC cells. Pulse-exposure of DRO81-1 cells for 3 days to either $1 \mu \mathrm{mol} / \mathrm{L}$ WGA, 250 $\mathrm{nmol} / \mathrm{L}$ WGB-MA, or $250 \mathrm{nmol} / \mathrm{L}$ WGB-DA irreversibly inhibited $80 \%$ clonogenic growth compared with untreated DRO81-1 cells (Fig 4).

\section{Withanolides suppressed activation of RET and Akt/mTOR pathway}

To elucidate the mechanism by which withanolides induce apoptosis and suppress MTC cell viability and growth, we evaluated by Western blot analysis the effects of these drugs on several key regulatory pathways and proteins in MTC cells, specifically activation of RET, the MAP kinase pathway (ERK1/2) and the Akt/mTOR pathway. Because alterations in protein expression in response to drug treatment may be both time- and concentrationdependent, we evaluated each drug at multiple doses (above and below $\mathrm{IC}_{50}$ levels) at 12 hours and at 24 hours treatment. Comparisons of expressions of these signaling proteins are shown for each withanolide drug in Fig 5. WGA, WGB-MA, and WGB-DA each demonstrated a consistent pattern related to their mechanistic effects on MTC signaling pathways. Each of these withanolides downregulated total and phospho-RET expression levels as well as total and phospho-mTOR expression levels and total and phospho-Akt levels. Downstream mTOR signaling was also effected in these withanolide-treated cells. Because mTOR modulates activity of downstream 4E-BP1 and p70S6kinase to regulate RNA translation and protein synthesis respectively, these proteins were evaluated in response to drug treatment. All 3 withanolides demonstrated inhibition of 4E-BP1, phospho-4E-BP1, p70S6kinase, and phospho-p706Skinase at 24 hours drug treatment with downregulation occurring at $500 \mathrm{nmol} / \mathrm{L}$ concentrations, which is at or below $\mathrm{IC}_{50}$ drug levels in DRO81-1 cells (Fig 5). ERK1/2 expression levels, however, were increased with drug treatment. Inhibition of both RET activity and the Akt/mTOR pathway occurred as early as 12 hours after treatment. WGD, which has the lowest potency in vitro and lacks the double bond in the A-ring (which conveys activity), demonstrated up-regulation of RET, Akt, ERK1/2, and the mTOR pathway, including 4E-BP1 and p70S6 kinase, which 
coincided with its lack of in vitro growth inhibition or induction of apoptosis (Fig 5,D). Finally, vandetanib and cabozantinib (clinical TKIs used in MTC) were evaluated for their effects on the same regulatory proteins in MTC cells (Fig 6). Both of these drugs inhibited phospho- and total RET expression levels at 24 hours, although significantly less robustly than noted with WGB-MA or WGB-DA withanolide treatment $(P<.01$ based on densiometry measurements of comparative protein bands). Interestingly, these targeted therapies did not inhibit mTOR or its downstream constituent p-70S6 kinase, although only at $3 \mu \mathrm{mol} / \mathrm{L}$ (10 times the $\mathrm{IC}_{50}$ level) did vandetanib slightly downregulate 4E-BP1 levels. Both TKIs markedly downregulated ERK1/2 and phospho-ERK expression levels while upregulating Akt expression levels in a dose-dependent manner.

\section{DISCUSSION}

For patients with unresectable or advanced MTC, a targeted therapy trial has been an approach of choice when such treatments are available. With cabozantinib completing its Phase III international trial and vandetanib's recent US Food and Drug Administration approval for use in MTC, these targeted agents are the most commonly used clinically. ${ }^{17}$ Outcomes from these studies, however, are modest at best and each of these drugs carry moderate toxicity profiles.

Natural products have played a significant role in the discovery and development of new anticancer agents, and represent a rich source of biologically active compounds. More than $80 \%$ of anticancer drugs introduced from January 1981 to October 2008 were natural products, semi-synthetic analogs, or synthetic compounds based on natural product pharmacophores. ${ }^{18}$ Withanolides are naturally occurring C-28-steroidal lactones built on an intact or rearranged ergostane scaffold in which C-22 and C-26 are appropriately oxidized to form a $\delta$-lactone ring on the 9 -carbon side chain. ${ }^{19}$ These compounds possess various biological activities in vitro and in vivo, including antimicrobial, radiosensitizing, antineoplastic, and cytotoxic effects. ${ }^{20-22}$ Although recent studies have demonstrated that WA suppresses the growth of human cancer cell lines in vitro and in vivo by causing apoptosis, ${ }^{15}$ the precise mechanism, including the signals and the pathways by which WA induces cell death, is not fully understood. Our group has demonstrated that WA inhibits MTC growth in vivo suppressing RET phosphorylation in the tumors as well as inhibiting calcitonin secretion. ${ }^{15}$ This compound did not result in weight loss or any clinical toxicity in vivo and has good oral bioavailability.

In the current experiments, we demonstrated that 3 novel withanolide compounds from the Physalis plant demonstrated potent activity against MTC cells in vitro with $\mathrm{IC}_{50}$ levels for acetylated analogs WGB-MA and WGB-DA in the mid-nanomolar range, comparable with the potency of targeted TKIs such as vandetanib and cabozantinib. Treatment of MTC with withanolides significantly irreversibly reduced clonogenic formation. Their mechanism of action for this antiproliferative effect involves several processes including induction of apoptosis, which was significantly enhanced with the acetylated analogs WGB-MA and WGB-DA and involved $>60 \%$ of cells at $1 \mu \mathrm{mol} / \mathrm{L}$ WGB-DA and $83 \%$ of cells at $3 \mu \mathrm{mol} / \mathrm{L}$ of drug. These compounds also affect cell-cycle arrest, with significant shift of cells into G2/M arrest, as reported with WA. ${ }^{21}$ 
Given the high apoptotic rate with treatment, we investigated several key regulatory proteins in MTC cells. The RET proto-oncogene is activated in MTC, leading to activation of the RAS/RAF/MEK/ERK signaling pathway. Mutant RET with representative MEN2A mutation at Cys-634 (RET-MEN2A) has been shown to activate PI3 kinase/Akt pathway. ${ }^{23}$ Furthermore, a recent study by Kouvaraki et al ${ }^{24}$ demonstrated that mTOR is highly active in MTC cells. Because mTOR signaling controls protein synthesis through regulation of translation initiation, identifying small molecules that target the mTOR pathway represents a promising target for investigational therapy in MTC patients. The present study shows that novel withanolides WGA, WGB-MA, and WGB-DA reduce both RET and Akt/mTOR activation in MTC cells with inhibition of mTOR RNA translation through knockdown of 4E-BP1. This mechanism is unique from TKIs like ventatenib or cabozantinib, which knock down RET and the MAP kinase pathway but do not affect the mTOR pathway. These TKIs upregulate Akt expression levels, which may explain in part why apoptosis is not seen as robustly in MTC cells with these therapies. These experiments are the first to identify this unique mechanism of action of withanolide compounds in MTC cells. Because the novel withanolide molecules WGA, WGB-MA, and WGB-DA work on different key regulatory pathways than TKIs in MTC cells, there may be a role for synergy or combination therapies of withanolides with these targeted TKIs. With similar mechanism to their parent molecule, WA, these novel natural withanolide analogs have potent anticancer activity in vitro, whereas WA itself has now progressed into a phase I clinical trial in India in patients with advanced osteosarcomas. ${ }^{25}$ Overall, these novel withanolides demonstrate excellent potency and anticancer activity in MTCs in vitro downregulating both RET and mTOR activity. Ongoing in vivo efficacy and toxicity studies will better evaluate their clinical potential.

\section{Acknowledgments}

Made possible by grant support from the National Institutes of Health (NIH-COBRE P20 RR015563 P.I. B. Timmermann) as well as from the Institute for Advancing Medical Innovation (PI: MS Cohen).

\section{References}

1. American Cancer Society Facts and Figures. 2010

2. Chong GC, Beahrs OH, Sizemore GW, Woolner LH. Medullary carcinoma of the thyroid gland. Cancer. 1975; 35:695-704. [PubMed: 1111937]

3. Cohen MS, Moley JF. Surgical treatment of medullary thyroid carcinoma. J Intern Med. 2003; 253:616-26. [PubMed: 12755957]

4. Sanders EM Jr, LiVolsi VA, Brierley J, Shin J, Randolph GW. An evidence-based review of poorly differentiated thyroid cancer. World J Surg. 2007; 31:934-45. [PubMed: 17431717]

5. Santoro M, Carlomagno F. Drug insight: Small-molecule inhibitors of protein kinases in the treatment of thyroid cancer. Nat Clin Pract Endocrinol Metab. 2006; 2:42-52. [PubMed: 16932252]

6. Mulligan LM, Kwok JB, Healey CS, Elsdon MJ, Eng C, et al. Germ-line mutations of the RET protooncogene in multiple endocrine neoplasia type 2A. Nature. 1993; 363:458-60. [PubMed: 8099202]

7. Santoro M, Fusco A. New drugs in thyroid cancer. Arch Bras Endocrinol Metabol. 2007; 51:85761.

8. Schlumberger M, Carlomagno F, Baudin E, Bidart JM, Santoro M. New therapeutic approaches to treat medullary thyroid carcinoma. Nat Clin Pract Endocrinol Metab. 2008; 4:22-32. [PubMed: 18084343] 
9. Sherman SI. Targeted therapy of thyroid cancer. Biochem Pharmacol. 2010; 80:592-601. [PubMed: 20471374]

10. Yokota Y, Bargagna-Mohan P, Ravindranath PP, Kim KB, Mohan R. Development of withaferin A analogs as probes of angiogenesis. Bioorg Med Chem Lett. 2006; 16:2603-7. [PubMed: 16513346]

11. Mishra LC, Singh BB, Dagenais S. Scientific basis for the therapeutic use of Withania somnifera (ashwagandha): a review. Altern Med Rev. 2000; 5:334-46. [PubMed: 10956379]

12. Misra L, Mishra P, Pandey A, Sangwan RS, Sangwan NS, Tuli R. Withanolides from Withania somnifera roots. Phytochemistry. 2008; 69:1000-4. [PubMed: 18061221]

13. He QP, Ma L, Luo JY, He FY, Lou LG, Hu LH. Cytotoxic withanolides from Physalis angulata L. Chem Biodivers. 2007; 4:443-9. [PubMed: 17372946]

14. Grover A, Shandilya A, Punetha A, Bisaria VS, Sundar D. Inhibition of the NEMO/IKKbeta association complex formation, a novel mechanism associated with the NF-kappaB activation suppression by Withania somnifera's key metabolite withaferin A. BMC Genomics. 2010; 11(Suppl 4):S25. [PubMed: 21143809]

15. Samadi AK, Mukerji R, Shah A, Timmermann BT, Cohen MS. A novel RET inhibitor with potent efficacy against medullary thyroid cancer in vivo. Surgery. 2010; 148:1228-36. [PubMed: 21134556]

16. Koduru S, Kumar R, Srinivasan S, et al. Notch 1 Inhibition by Withaferin-A: A Therapeutic Target against Colon Carcinogenesis. Mol Cancer Ther. 2010; 9:202-10. [PubMed: 20053782]

17. Sagar, S. NCT00704730. An International, Randomized, Double-Blinded, Phase 3 Efficacy Study of XL184 Versus Placebo in Subjects With Unresectable, Locally Advanced, or Metastatic Medullary Thyroid Cancer. 2010. www.clinicaltrials.gov

18. Cragg GM, Grothaus PG, Newman DJ. Impact of natural products on developing new anti-cancer agents. Chem Rev. 2009; 109:3012-43. [PubMed: 19422222]

19. Gunasekera SP, Kinghorn AD, Cordell GA, Farnsworth NR. Plant anticancer agents. XIX Constituents of Aquilaria malaccensis. J Nat Prod. 1981; 44:569-72. [PubMed: 7320738]

20. Samadi AK, Tong X, Mukerji R, Zhang H, Timmermann BN, Cohen MS. Withaferin A, a cytotoxic steroid from Vassobia breviflora, induces apoptosis in human head and neck squamous cell carcinoma. J Nat Prod. 2010; 73:1476-81. [PubMed: 20726569]

21. Samadi A, Loo P, Mukerji R, Timmermann BN, Cohen MS. A novel HSP90 modulator with selective activity against thyroid cancers in vitro. Surgery. 2009; 146:1196-207. [PubMed: 19958949]

22. Stan SDHE, Warin R, Singh SV. Withaferin A causes FOXO3a- and Bim-dependent apoptosis and inhibits growth of human breast cancer cells in vivo. Cancer Res. 2008; 68:7661-9. [PubMed: 18794155]

23. Segouffin-Cariou C, Billaud M. Transforming ability of MEN2A-RET requires activation of the phosphatidylinositol 3-kinase/AKT signaling pathway. The Journal of Biological Chemistry. 2000; 275:3568-76. [PubMed: 10652352]

24. Kouvaraki MA, Liakou C, Paraschi A, Dimas K, Patsouris E, Tseleni-Balafouta S, Rassidakis GZ, Moraitis D. Activation of mTOR signaling in medullary and aggressive papillary thyroid carcinomas. Surgery. 2011; 150:1258-65. [PubMed: 22136849]

25. Gota, V. A phase I safety and pharmacokinetics study of Withaferin-A in patients with advanced stage high grade osteosarcoma. 4th International Conference on Drug Discovery and Therapy; Feb 2012; Abstract published online http://icddt.com/abstracts/SL/SL-01-29-2012_083_Vikram $\% 20$ Gota.htm 




Fig 1.

Comparative chemical structures of withanolides and tyrosine kinase inhibitors tested. Chemical structures are included for WA, WGA, WGA-TA, WGD, WGB-MA, WGB-DA, axitinib, vandetanib, cabozantinib, and 17-AAG. 

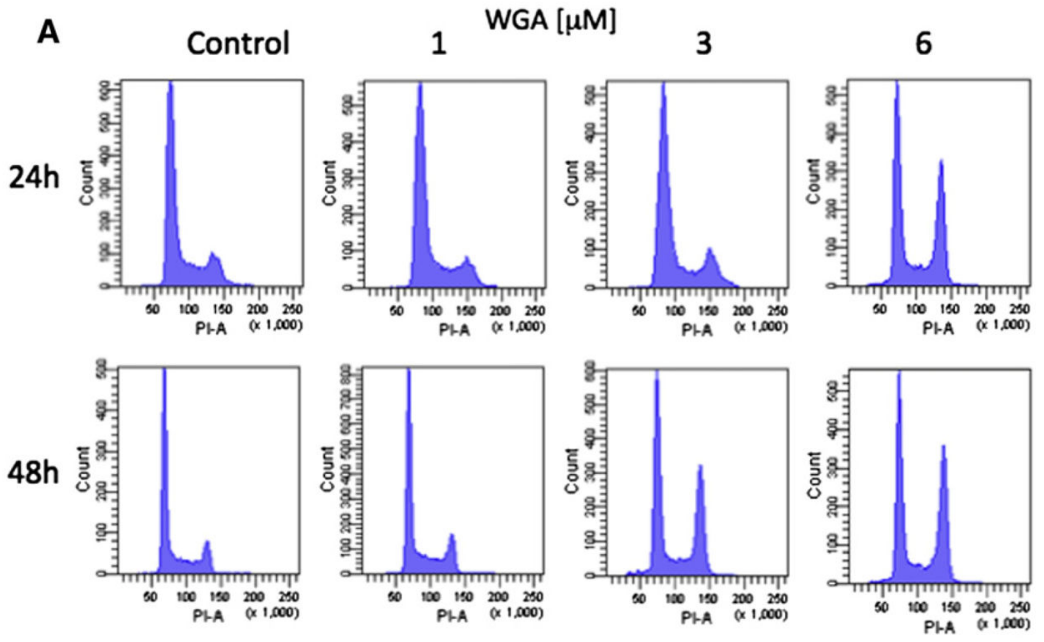

B
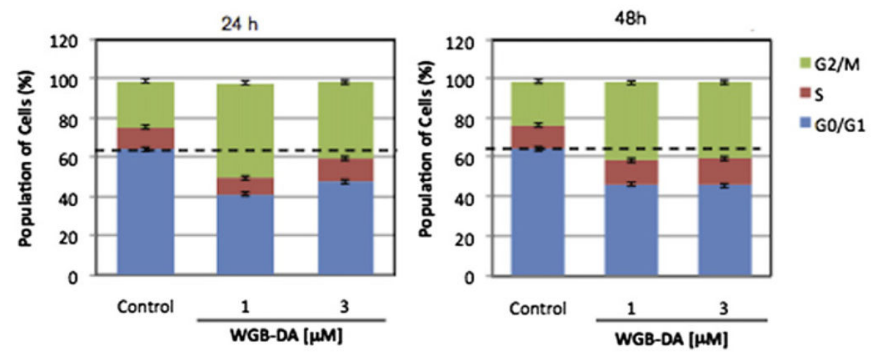

Fig 2.

Novel withanolides induce G2/M cell-cycle arrest in MTC cells. A, PI staining by flow cytometry of DRO81-1 cells exposed to WGA cells at concentrations of 1-6 $\mu \mathrm{mol} / \mathrm{L}$ for 24 and 48 hours. Of note there is a significant shift in cell-cycle arrest with an increase in the size of the G2/M peak with increasing dose of WGA treatment at 48 hours. $B$, Bar graphs with quantitative shifts in the cell cycle of DRO81-1 cells treated with either WGA, WGBMA, or WGB-DA for 24 or 48 hours. The shift was significant to G2/M arrest with withanolide treatment. (Color version of figure is available online.) 

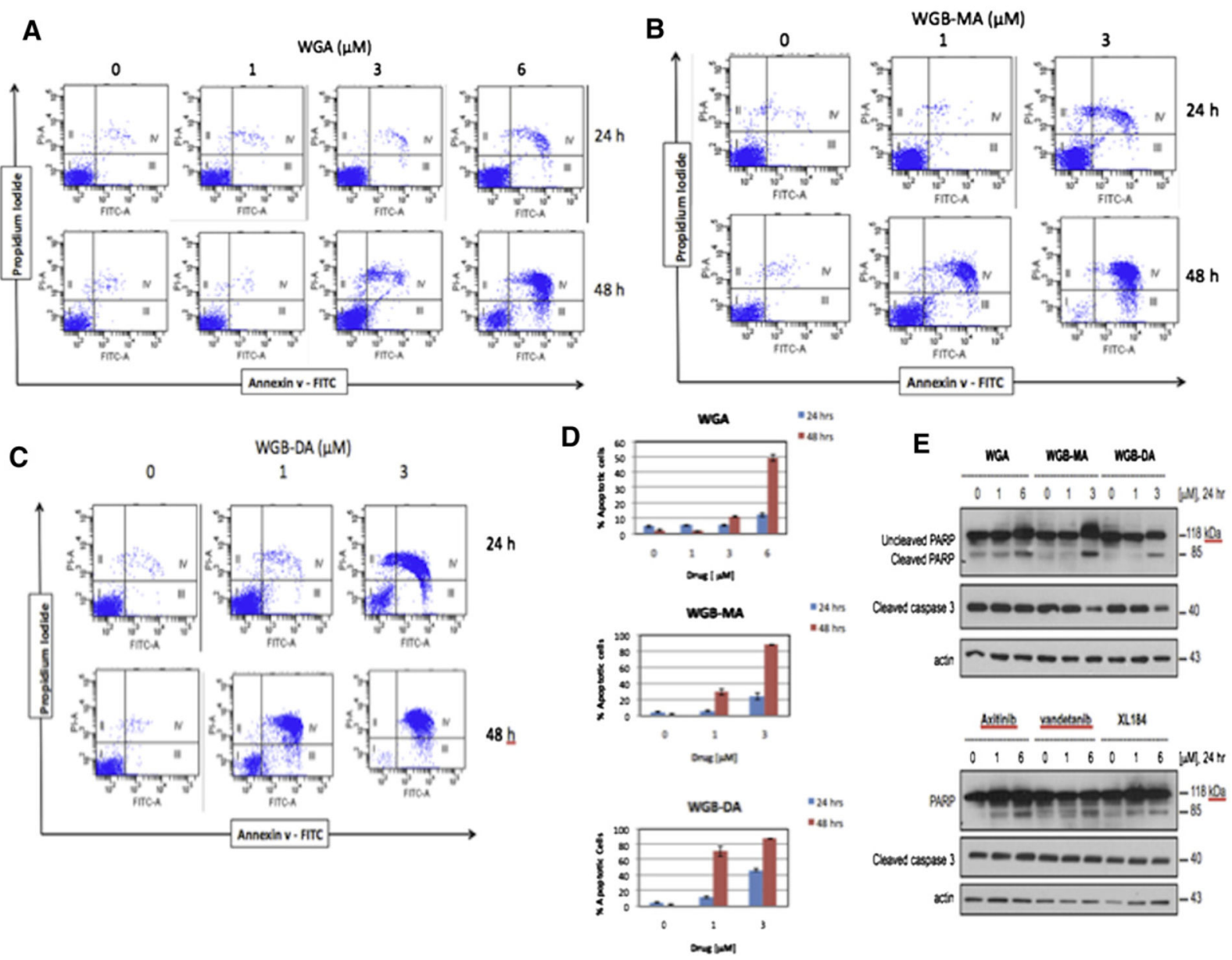

Fig 3.

Novel withanolides induce apoptosis in MTC cells. Dot-blot flow cytometry data for costaining with Annexin V and PI after treatment with different concentrations of 3 novel withanolides, $(A)$ WGA, $(B)$ WGB-MA, and (C) WGB-DA. Significant apoptosis was observed at $6 \mu \mathrm{mol} / \mathrm{L}$ WGA, and $1 \mu \mathrm{mol} / \mathrm{L}$ WGB-MA, and WGB-DA. $D$, Quantitative apoptosis of MTC cells treated with these withanolides for 24 and 48 hours. More than $80 \%$ of cells were gated toward apoptotic cell death with only $3 \mu \mathrm{mol} / \mathrm{L}$ exposure of WGB-DA. $E$, Confirmation of apoptosis by Western Blot analysis, demonstrating activation of caspase 3 and cleavage of PARP in a dose-dependent manner, starting at $1 \mu \mathrm{mol} / \mathrm{L}$ of withanolide drug. This effect is less robust with the TKI drug compounds at similar concentrations. (Color version of figure is available online.) 


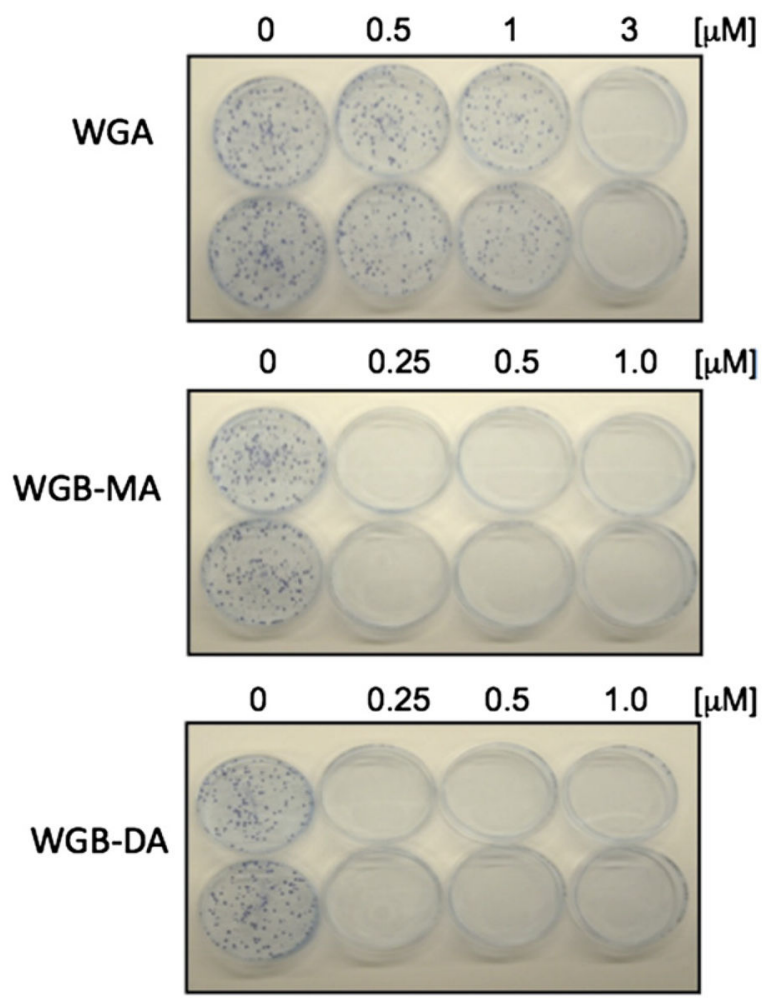

Fig 4.

Novel withanolides inhibit clonogenic growth in MTC cells. Inhibition of clonogenic growth formation by WGA at $1 \mu \mathrm{mol} / \mathrm{L}$ drug concentration; WGB-MA and WGB-DA inhibited growth at only $250 \mathrm{nmol} / \mathrm{L}$ concentrations, consistent with $\mathrm{IC}_{50}$ values. This assay suggests that the anticancer effects of withanolides on MTC cells are longer term and not easily reversible. (Color version of figure is available online.) 

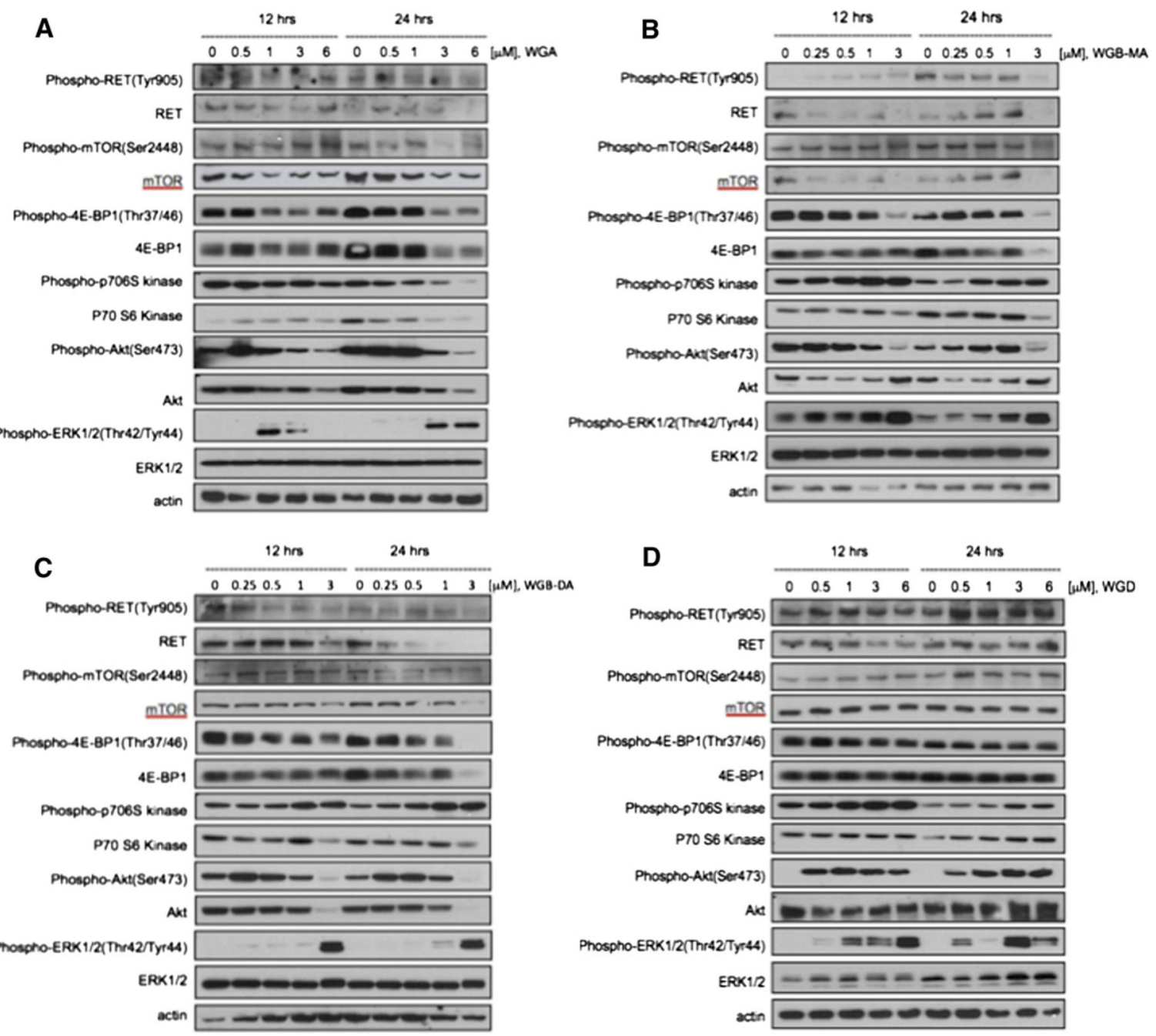

Fig 5.

Comparison of the effects of withanolide drug treatment on the expression of key regulatory proteins involved in MTC cells using Western Blot Analysis. (A) WGA effects on protein expression. (B) Expression changes from WGB-MA. (C) WGB-DA mechanistic actions. $(D)$ WGD actions. The active withanolides WGA, WGB-MA, and WGB-DA down-regulated RET, p-RET, mTOR, p-mTOR, 4E-BP1, and Akt expression in a dose-dependent manner. These data indicate that withanolides not only knock down RET activity, but also inhibit the Akt/mTOR regulatory pathway, which may account in part for their robust induction of apoptosis in MTC cells. WGD was inactive on MTS assay owing to loss of the double bond in the A-ring of the structure. This compound upregulated mTOR, Akt, and ERK as well as RET activity. 

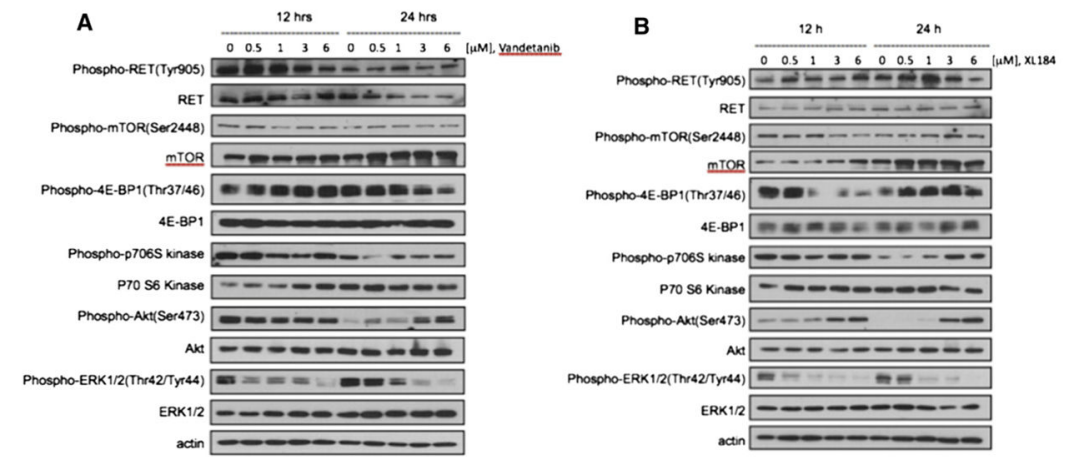

Fig 6.

Comparison of the effects of vandetanib and cabozantinib drug treatment on the expression of key regulatory proteins involved in MTC cells using Western blot analysis. Both TKIs were evaluated for their effects on similar regulatory proteins studied in Fig 5. Withanolides differ from TKIs in their mechanism of action in that the 2 TKIs tested do not inhibit the $\mathrm{Akt} / \mathrm{mTOR}$ signaling pathway, but rather downregulate RET activity and the MAP kinase pathway. These differences in action suggest a potential for synergy or combination therapy. 
Table

$\mathrm{IC}_{50}$ levels of novel withanolides and tyrosine kinase inhibitors

\begin{tabular}{llcl}
\hline & \multicolumn{3}{c}{ IC $_{\mathbf{5 0}}(\boldsymbol{\mu m o l} / \mathbf{L})$} \\
\cline { 2 - 4 } Compounds & DRO81-1 & MTC-TT & TPC-1 \\
\hline WA & 1.09 & 2.76 & 0.48 \\
WGA & 2.85 & 2.17 & 0.29 \\
WGD & 5.84 & 17.0 & 4.39 \\
WGB-MA & 0.96 & 1.31 & 0.077 \\
WGB-DA & 0.58 & 0.86 & 0.030 \\
WGA-TA & 0.27 & 1.03 & 0.039 \\
Axitinib & 1.18 & 0.78 & 0.95 \\
Vandetanib & 2.38 & 0.25 & 0.27 \\
XL184 & 2.64 & 0.36 & 0.16 \\
17-AAG & 0.012 & 0.024 & 0.015 \\
\hline
\end{tabular}

